\title{
TRAINING AND THE TWO WAY MEMORY EFFECT IN COPPER BASED SHAPE MEMORY ALLOYS
}

\author{
R. STALMANS, J. van HUMBEECK and L. DELAEY \\ Department of Metallurgy and Materials Engineering, Katholieke Universiteit Leuven, De Croylaan 2, \\ B-3001 Heverlee, Belgium
}

\begin{abstract}
Experimental results giving information about the mechanisms of the two way memory effect are presented. It is shown that local martensite stabilization and true plastic deformations have to be considered as undesirable side-effects of training. The experimental results also indicate that the macroscopic two way memory behaviour is, on a microstructural level, associated with a reproducible and stable martensite formation path. The higher the amount of transformation cycles the better becomes the stability and reproducibility. In accordance with the stable path is that the two way memory effect can withstand considerable forces during the forward transformation (the two way memory can do work during cooling). Another consequence is that the maximum attainable two way memory diminishes when the training procedure is preceded by thermal transformation cycles.
\end{abstract}

\section{INTRODUCTION}

In 1972, Tas et al [1] proposed the term "two way memory effect" (abbreviated to TWME) to designate the spontaneous reversible shape effect during cooling and heating, which was observed after particular thermomechanical procedures. Since that time many papers on the TWME, the thermomechanical procedures and the mechanisms of the TWME have been published. The thermomechanical procedures are mostly based on the repetition (therefore described as "training") of thermomechanical cycles through the transformation region. Two mechanisms for the TWME have been proposed, either based on residual stress fields or on locally retained martensite.

Perkins [2] explained in 1974 the TWME as a result of a macroscopic non-uniform residual stress field. From this it was concluded that a non-uniform plastic deformation was a necessary prerequisite to obtain a TWME. Later on, however, it was shown that non-uniformity was an unduly limiting criterion [3]. In accordance with the observation of dislocation structures generated by training [4,5], the TWME was then attributed to the microscopic residual stress fields around those dislocation structures. It was proposed [6,7] that those residual stress fields favour the nucleation and the beginning of growth of some preferential variants, and at the same time the residual stresses are relaxed by the accompanying shape change. During further cooling those preferential variants grow without any assistance, thus causing the TWME. Based on the assumption that those dislocations are linked with plasticity, a plastic deformation was still considered as being essential for the TWME in [4] and [5].

A second mechanism is based on the local stabilization of martensite, retained above the original $A_{f}$ temperature $[4,9]$. The growth of this locally stabilized preferential martensite is responsible for the 
TWME. This mechanism also necessitates a residual deformation of the hot shape since the retransformation to the high temperature phase (further called austenite) is incomplete.

Both mechanisms only favour the beginning of transformation. If that is the case, the spontaneous growth of the preferential variants can easily be prevented by an external applied opposing stress. Consequently, based on those mechanisms, it is widely believed that the TWME cannot withstand important opposing stresses during cooling through the transformation region [9]. However, conclusive experimental evidence for the proposed mechanisms and for the deduced conclusions is still lacking. In this paper experimental results are shown which are in contradiction with the above proposed "fixed" ideas about the TWME.

Information about the experimental details can be found in [10].

\section{RESULTS}

\section{Training and the TWME}

In this paragraph some of the results presented in [10] are summarized. Figure 1 shows the general training cycle in a strain-temperature diagram. The sample is heated to a maximum temperature $T_{A}$ (above Af), followed by increasing the stress from zero to the training stress. Afterwards the sample is cooled to a minimum temperature $\mathrm{T}_{\mathbf{C}}$ (below Mf) while the stress is kept constant. The stress is then decreased to zero and the sample is reheated to the initial temperature $\mathrm{T}_{\mathrm{A}}$. Each training cycle was followed by a thermal cycle to investigate the two way memory effect. The transformation strain induced by the stressed cooling is designated by SATE : stress-âssisted transformation effect.

The magnitude of the TWME was found to be determined directly by the number of training cycles and the magnitude of SATE :

* by increasing the number of training cycles (and thus by repeating the SATE) the TWME approaches more and more the nearly constant SATE (figure 2);

* for a fixed number of training cycles, it was found that the TWME is linearly related to the SATE.

The latter result shows that the training stress and the TWME are only indirectly related. The training stress determines the degree of preferential martensite formation during stressed cooling and thus the SATE and this SATE determines directly the TWME.

It was also found that plastic deformation (1) and local martensite stabilization (2) have to be considered as being mainly side-effects of "overtraining" instead of being essential to the TWME.

(1) As a consequence of the indirect relationship, the TWME saturates at a rather low training stress (figure 3). A further increase of the training stress, which can be called "over"stressing, results in a strong increase of the plastic deformation of the hot shape without an increase of the TWME. This contradicts with the opinion that a considerable plastic deformation is a necessary prerequisite for the TWME.

(2) The TWME shows also a maximum as a function of the number of training cycles. The number corresponding with this maximum, which was for the studied alloy about 30, was found to be independent of the training stress. Further training cycles, which can be called "over"cycling, resulted in a decrease of the TWME (figure 2). This was found to be mainly due to an increasing amount of locally stabilized martensite, retained above the maximum training temperature $T_{A}$. The reversion of the locally stabilized martensite by overheating has a negligible effect on the cold shape. This shows that the spontaneous growth of the locally stabilized martensite is not the underlying mechanism of the TWME. 


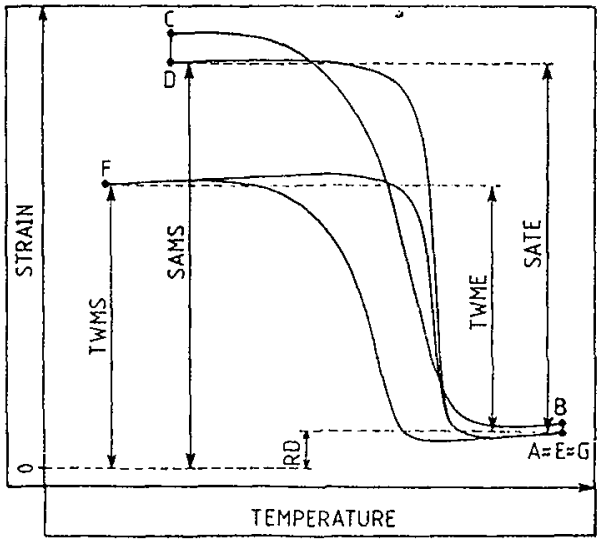

Fig.1. The nth training and subsequent thermal cycle. TWME : two way memory effect, SATE : stress assisted transformation effect, $\mathrm{RD}$ : residual deformation of the austenitic shape, TWMS : two way martensitic strain, SAMS : stress assisted martensitic strain.

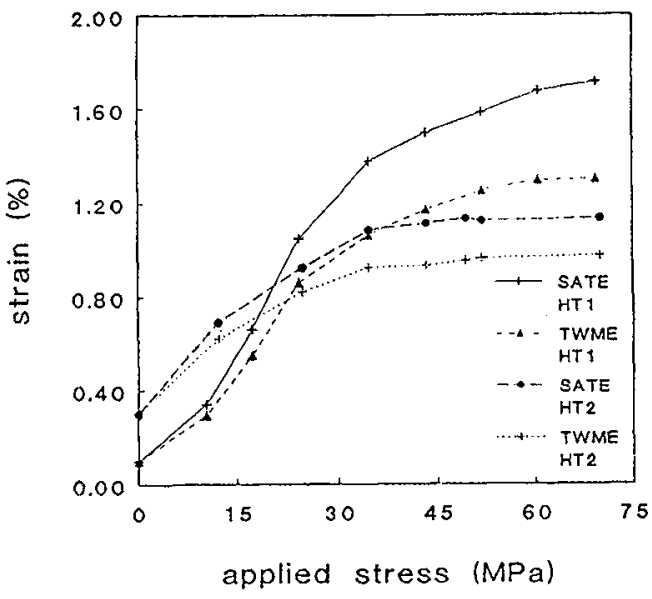

Fig.3. The two way memory effect (TWME) and the stress assisted transformation effect (SATE) as a function of the applied training stress. (training cycle $30 ;$ HT1 : heat treatment 1 ; HT2 : hcal treaument 2)

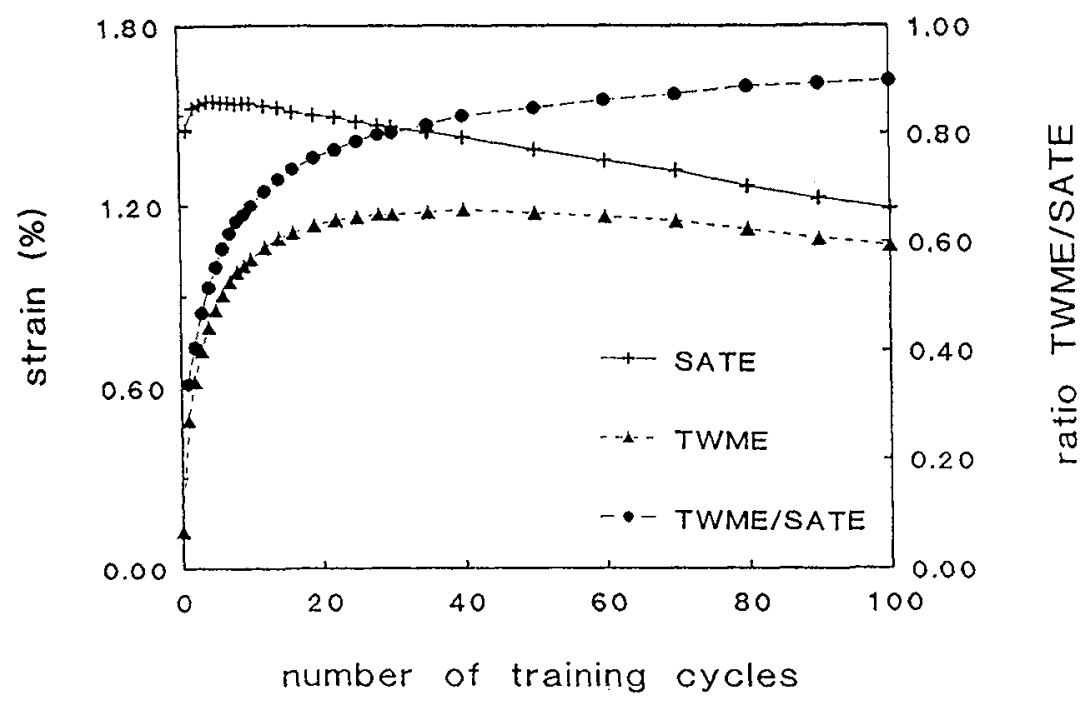

Fig.2. The evolution of the SATE, TWME and the ratio TWME/SATE during training is represented. (heat treatment 1, training stress $43.4 \mathrm{MPa}$ ) 


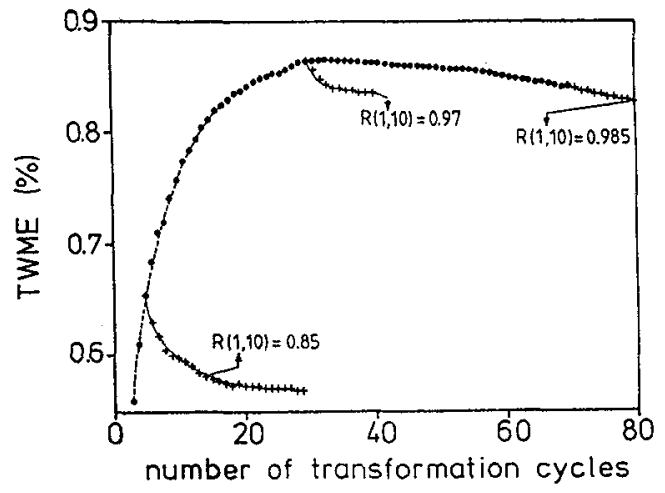

Fig.4. The evolution of the TWME during training $(\rightarrow-)$ and subsequent thermal cycling $(-)$. The reproducibility increases with the number of training cycles. (heat treatment 1 , training stress $34.7 \mathrm{MPa}$ )

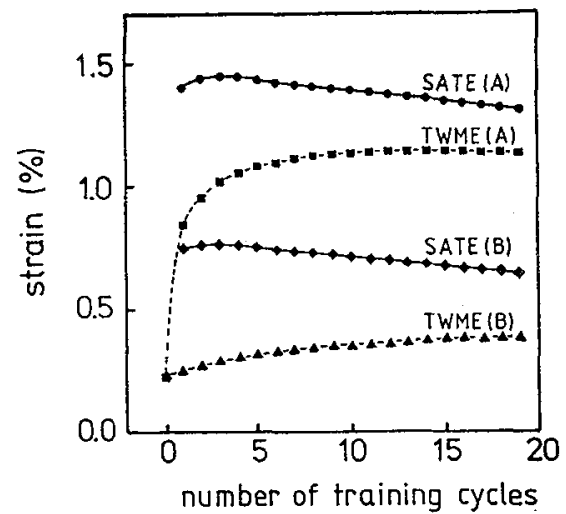

Fig.5. The influence of prior thermal cycling on the evolution of the SATE and TWME during training. (A: 0 prior thermal cycles, B : 70 prior thermal cycles)

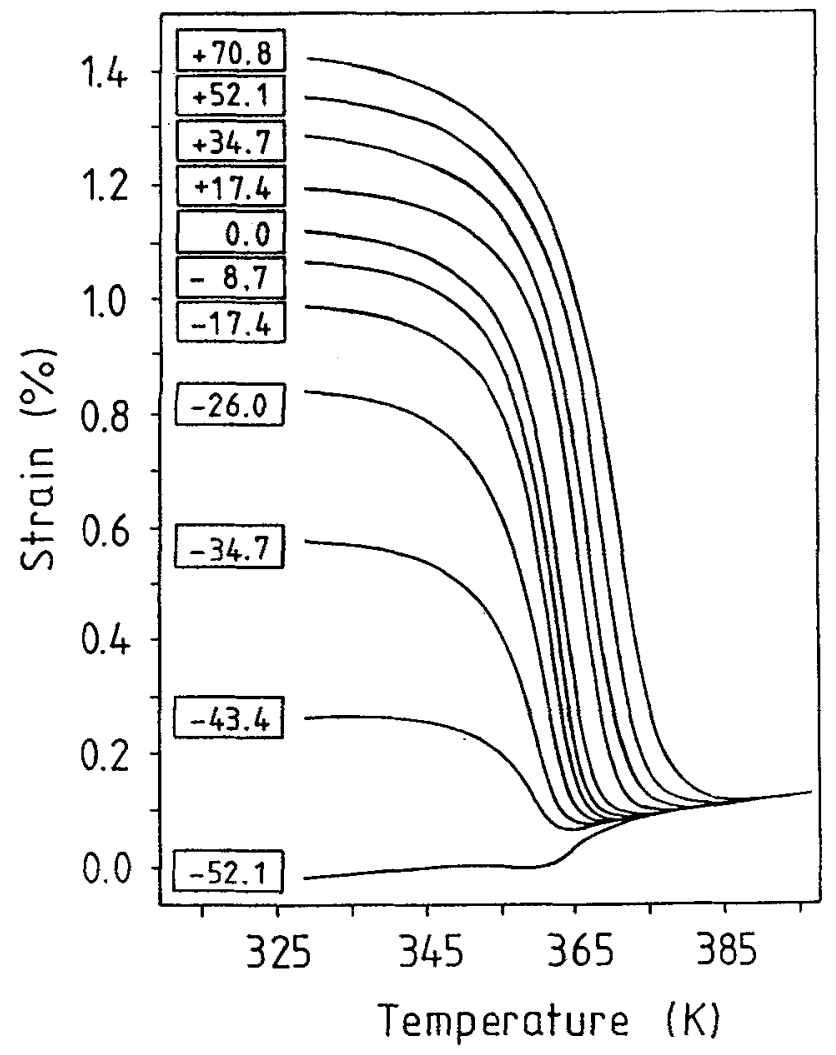

Fig.6. Strain-temperature curves during cooling at different applied stresses of a trained specimen (heat treatment 1 - training : 30 cycles, training stress $34.7 \mathrm{MPa}-100$ thermal cycles). The applicd stresses are given in the rectangulars. 
Reproducibility and the number of training cycles

We define the reproducibility $R(i, j)$ of the TWME as :

$R(i, j)=\frac{T W M E(j)}{T W M E(i)}$

with $\mathrm{i}$ and $\mathrm{j}$ standing for respectively the $\mathrm{i}^{\text {th }}$ and $\mathrm{j}^{\text {th }}$ thermal cycle after training.

Reproducibility and degradation of the TWME are thus considered as mutual opposites.

Specimens were trained for different number of training cycles, followed by thermal cycling to investigate the reproducibility. Figure 4 shows that the reproducibility of the TWME increases at a declining rate during training. A nearly perfect reproducibility $(R(1,10): 0.96-0.98)$ is obtained after about 30 training cycles.

\section{Thermal cycling before training}

Samples have been thermally cycled under no load before applying the training procedure. Some examples showing the influence of prior thermal cycling are given in figure 5.

Two general conclusions can be derived from the experiments.

1. When the training routine is preceded by thermal cycles, a smaller TWME is obtained. Summarized, the higher the number of prior thermal cycles, the smaller becomes the attainable TWME.

2. The same can be said about the SATE. The lowering of the SATE by prior thermal cycling indicates that prior thermal cycling increases the resistance to preferential martensite formation.

\section{The TWME and opposing stresses - Stability of the TWME}

Stability of the TWME is defined here as the resistance to a decrease of the TWME when an opposing stress is applied during cooling.

A sample was subjected to 30 training cycles followed by 100 thermal cycles. From the previous paragraph it is known that this procedure results in a highly reproducible TWME ( $R(99,100) \approx 1)$. Subsequently the sample was cycled through the transformation region with different applied stresses, as well tensile, as compressive (and thus opposing the TWME) stresses. Part of the results are shown in figure 6. From the experiments, several important conclusions can be deduced.

1. In contradiction to what is generally accepted, the TWME can withstand considerable opposing stresses : an opposing stress of $52 \mathrm{MPa}$ during cooling is required to suppress the TWME completely.

2. Consequently, the TWME can do work. The maximum work output, which was obtained at an opposing stress of about $-25 \mathrm{MPa}$, was about $0.025 \mathrm{~J} / \mathrm{g}$.

3. The $M_{S}$ and $A_{f}$ transformation temperatures are shifted downwards with increasing opposing stresses.

\section{GENERAL DISCUSSION}

The influence of the number of training cycles on the magnitude and the reproduciblity of the TWME shows a striking resemblance : the reproducibility and the magnitude of the TWME both increase at a declining rate during training and reach a saturation value after about 30 training cycles.

Reproducibility and stability of the TWME are found to be closely related as shown by the following. After a sufficient number of training cycles, a highly reproducible TWME is obtained. This reproducible TWME can withstand considerable opposing stresses during cooling and hence corresponds with a highly stable TWME. The reproducibility of the TWME is the macroscopic consequence of the microscopic reproducibility of the transformation path [11]: in subsequent cycles, the same variants are formed in the 
same sequence during cooling. In the same way, the stability of the TWME is the consequence of a stable transformation path, which is generated by training. The martensite variants corresponding with this path even grow when opposing stresses are applied. Since the transformation is thermoelastic, the growth of those stable variants must be energetically favoured to other variants. This conclusion is in contrast with the two in the introduction proposed mechanisms, which are both based on spontaneous growth.

A plausible and energetically based explanation is that those variants are growing in which the dislocation structures have the lowest energy, as already suggested by Sade et al [12]. From this a general mechanism for the TWME can be deduced : dislocations are generated by transformation cycling (training) and consequently the martensite transformation path is becoming more and more stabilized.

This mechanism explains the previous experimental results.

* During training the TWME resembles more and more the SATE since the preferential transformation path responsible for he SATE becomes more and more stabilized.

* The reproducibility is a logic consequence of the stability since a reproducible transformation path is a logical outcome of a stable transformation path.

* The higher the number of thermal transformation cycles the better becomes the stability of the "trained" self-accommodating transformation path. Consequently there is a higher resistance to the preferential martensite formation. This explains the lowering of the SATE by prior thermal cycling. In other words, a thermally cycled specimen cannot be "re"trained.

\section{ACKNOWLEDGEMENTS}

R. Stalmans and J. Van Humbeeck are respectively Research Assistant and Research Associate of the NFWO (National Fund for Scientific Research, Belgium).

\section{REFERENCES}

1. H.Tas, L. Delaey and A. Deruyttere, J.Less-Common Met. 28, 141 (1972)

2. J. Perkins, Scripta metall. 8, 1469 (1974).

3. R. J. Wasilewski, Scripta metall. 9, 417 (1975).

4. J. Perkins and R. O. Sponholz, Metall. Trans. 15A, 313 (1984).

5. D. Rios-Jara and G. Guenin, Acta metall. 35, 109 (1987).

6. G. Guenin, in : The Mart. Trans. in Sci. and Techn., Ed. E. Hornbogen and E. Jost, DGM Informationsgesellschaft, Oberursel, 39 (1989).

7. L. Contardo and G. Guenin, Acta metall. mater. 38, 1267 (1990).

8. A. Nagasawa, K. Enami, Y. Ishino, Y. Abbe and S.Nenno, Scripta metall. 11, 225 (1977)

9. J. Perkins and D. Hodgson, in : Engineering Aspects of Shape Memory Alloys, Ed. T. W. Duerig, K. N. Melton, D. Stöckel and C. M. Wayman, Butterworth-Heinemann, London, 195 (1990).

10. R. Stalmans, J. Van Humbeeck and L. Delaey, accepted for publication in Acta metall. mater.

11. L. Delaey and J. Thienel, in : Shape Memory Effects in Alloys, Ed. J. Perkins, Plenum Press, New York, 341 (1975).

12. M. Sade, A. Hazarabedian, A. Uribarri and F. C Lovey, Proc. Int. Conf. Solid. Phase. Transf., Ed. G. W. Lorimer, Cambridge, England, 279 (1987). 